\title{
Impact of Customers Flexibility in Heat Pumps Scheduling for Demand Side Management
}

\author{
Marco Pau, Jochen L. Cremer, Ferdinanda Ponci, Antonello Monti \\ Institute for Automation of Complex Power Systems \\ E.ON Energy Research Center - RWTH Aachen University \\ Mathieustrasse 10, 52074 Aachen, Germany \\ Email: [mpau, jcremer, fponci, amonti]@eonerc.rwth-aachen.de
}

\begin{abstract}
In the smart grid scenario, Demand Response (DR) and Demand Side Management (DSM) programs are considered as strategic to obtain a more efficient operation of the grid. The flexibility given by the final customers plays a key role to unlock the potential benefits offered by the application of these schemes. A classical example of flexible load that can be exploited for DR and DSM purposes is the electric heat pump. This paper aims at evaluating the main factors affecting the flexibility available in the management of electric heat pumps for space heating. The performed analysis allows identifying some indexes to quantify the available flexibility and highlights how the thermal comfort requirements of the customers affect the provided level of flexibility. Sample simulations show the impact of these flexibility terms on the results of a DSM program designed for power peak shaving at grid level. The possible use of the defined indexes for sorting the customers flexibility and for estimating the potential benefits offered by the adopted DSM scheme is also investigated and discussed.
\end{abstract}

\section{INTRODUCTION}

In future smart grids, the diffusion of Distributed Generation (DG) and other Distributed Energy Resources (DERs) will significantly change the way in which electric grids must be operated [1]. While allowing a sustainable and environmentally friendly generation of power, Renewable Energy Sources (RES) are highly intermittent and their fluctuations have to be duly compensated in order to guarantee the needed balance between generated and consumed power. Moreover, their production depends on the weather conditions, and thus large generation can be present also when not needed by the close loads, leading to reverse power flows, voltage rises and, in extreme cases, to the curtailment of their generated power.

On the other side, the characteristics of the power demand are also expected to radically change in the near future. In particular, the electrification of the transportation sector, with the ongoing diffusion of hybrid and electric vehicles, and the electrification of the building heating systems, through the installation of electric heat pumps, is likely to determine important modifications on the levels and shape of the power consumption patterns [2]. In such a complex scenario, new solutions are increasingly required to ensure the reliable

(c) 2017 IEEE. Personal use of this material is permitted. Permission from IEEE must be obtained for all other users. DOI: 10.1109/EEEIC.2017.7977681. Publisher version: http://ieeexplore.ieee.org/ document/7977681/ operation of the power system and to pursue higher energy efficiency targets.

A solution for supporting the efficient management of future smart grids is to exploit the flexibility available at the customer side. Customer flexibility can be given by loads whose operation can be interrupted, postponed or scheduled. The amount of controllable power and the time for which the operation of the customer loads can be delayed are possible indicators of the level of flexibility allowed by the end-user. Demand Side Management (DSM) and Demand Response (DR) programs are specific schemes conceived to achieve, to some extent, directly or indirectly, the intelligent control and management of the customers flexibility [3]. A large variety of DSM and DR mechanisms can be found in the literature, based on different market approaches or targeting different technical objectives [4]-[6]. In general, DSM programs can be classified in two main categories [7]:

- price-based programs: they try to induce a change in the consumption profile of the customers by raising the price of the energy during peak hours and offering energy at lower prices during off-peak hours.

- incentive-based programs: they permit the direct control, scheduling or curtailment of specific appliances allowed by the end-user, who is rewarded through special incentives in his tariff.

Even though there are several challenges still preventing the diffusion of DSM programs in many countries (mainly due to regulatory barriers), the potential benefits arising from the activation of these schemes are commonly recognized [8]. Among the different residential customer appliances, electrothermal devices used for space heating are particularly suitable for being used in DSM schemes [9]. Their suitability mainly derives from the thermal storage properties of the buildings, which leads to relatively slow thermal dynamics and allows facing possible curtailments or interruptions of the electrothermal device with no (or a limited) discomfort for the building occupants. Due to the already mentioned process of electrification of the heating systems (promoted in several countries to fulfil energy efficiency targets in the residential sector), the design of DSM solutions aimed at the management of such heating systems has become a very hot research topic in the last years. Several works can be found dealing with 
the thermal modeling of the system, proposing management strategies bringing economic savings for the customers (e.g. under price-based DSM programs), investigating the impact brought by the installation of additional thermal storage, or evaluating the technical benefits achievable, from the utility perspective, under different DSM mechanisms [10]-[14].

In this paper, an optimization algorithm for the day-ahead scheduling of electric heat pumps is first presented. The objective of the algorithm is to define a smart scheduling of the heat pump for achieving power peak shaving at grid level while providing the desired thermal comfort to the customers. Then, the focus of the paper is on the evaluation of the customers flexibility and of its impact on the design and results of the DSM strategy. The goal is twofold: 1) to understand how the customer flexibility is affected by different parameters, in order to infer what optimization results can be expected when having different parameter values; 2) to find possible indexes and criteria for ranking the flexibility level of the customers; this can be useful, for example, to define accordingly the rewards for the customers in an incentive-based scheme scenario, but also to estimate the potential benefit brought by the DSM scheme and to further refine its design.

\section{Heat Pumps Management Algorithm}

The heat pump management algorithm here presented is an optimization algorithm aimed at performing a centralized dayahead scheduling of the heat pumps for $n$ houses downstream a given substation. The detailed description and presentation of the algorithm can be found in [15]; here, for the sake of completeness, a concise summary of the adopted thermal model, of the considered constraints and of the main features of the algorithm is reported. The goal of the optimization is to minimize the power peak consumption over the day at the substation. This is a typical requirement for the utilities, since a power peak reduction leads to flatten the power profile along the day (allowing a more efficient operation of the grid), brings economic advantages at system level (by avoiding the use of more expensive generation units for covering the power peak and reducing the need for spinning reserve), and allows postponing reinforcement investments in case of grids operated close to their limits [8].

The objective function of the optimization algorithm is a linearised version of a quadratic function, through which the minimization problem can be converted into a Mixed Integer Linear Programming (MILP) formulation. The objective function considers as starting point a daily profile of expected power at the substation given by the aggregation of all the loads except the heat pumps (which can be obtained using statistical and/or historical data). Then it tries to allocate the operation of the heat pumps in the time slots with lower power consumption. Calling $\Delta E_{b, t}$ the generic $b$-th discrete energy box at time $t$, and $\alpha_{b}$ the corresponding weight (increasing for growing values of the power associated to the energy box), the objective function is expressed as:

$$
\underset{y_{h, t}}{\operatorname{minimize}} \sum_{t} \sum_{b} \alpha_{b} \Delta E_{b, t}
$$

From (1) it is possible to see that the power at the time slot $t$ has been discretized through the energy blocks $\Delta E_{b, t}$. Since energy blocks related to increasing levels of power are associated to increasing values of the weight $\alpha_{b}$, the algorithm automatically tries to allocate the operation of the heat pumps in the time slots with lower power consumption.

The optimization process also considers the constraints related to the thermal comfort to be provided to the customers. In particular, in the conceived DSM scheme, it is assumed that the customers can define the temperature they desire to have at home during the day, together with a certain tolerance band around this reference temperature. Such tolerance band determines the lower and the upper temperature boundaries $\Gamma_{h, t}^{L B}$ and $\Gamma_{h, t}^{U B}$, respectively, for each time slot $t$ and house $h$. The thermal comfort constraint resulting in the optimization process is thus:

$$
\Gamma_{h, t}^{L B} \leq T_{h, t}^{I N} \leq \Gamma_{h, t}^{U B} \quad \forall h, t
$$

where $T_{h, t}^{I N}$ is the indoor temperature of house $h$ at time $t$. Such constraint leads the optimization algorithm to choose a heat pump scheduling that minimizes the power peaks in the grid while guaranteeing at each time the thermal comfort required by the customers. In addition to (2), the following boundary constraints is also considered:

$$
T_{h, 0}^{I N}=\Gamma_{h}^{I N I} \quad \forall h
$$

where $\Gamma_{h}^{I N I}$ is the starting temperature value for house $h$ and $T_{h, 0}^{I N}$ is the corresponding indoor temperature value at $t=0$.

The temperature profiles included in the constraints (2) are obtained through the thermal model of the system. A simplified thermal model has been used to keep the linear formulation in the optimization process. The indoor temperature at time $t$ has been modelled as:

$$
T_{h, t}^{I N}=T_{h, t-1}^{I N}+\frac{\Delta t}{\mu_{h}^{H S} \gamma^{A R}}\left(Q_{h, t}^{H P}-Q_{h, t}^{L S}\right) \quad \forall h, t
$$

where $\mu_{h}^{H S}$ is the indoor air mass of house $h, \gamma^{A R}$ is the air heat capacity, $\Delta t$ is the discrete time step used for the optimization process, and $Q_{h, t}^{H P}$ and $Q_{h, t}^{L S}$ are the heat flow coming from the heat pump and the heat losses from the house to the outdoor environment, respectively.

The heat losses $Q_{h, t}^{L S}$ depend on a heat loss factor $\kappa_{h}^{H S}$ and on the difference between indoor and outdoor temperature $\Gamma_{h, t-1}^{O T}$ :

$$
Q_{h, t}^{L S}=\kappa_{h}^{H S}\left(T_{h, t-1}^{I N}-\Gamma_{h, t-1}^{O T}\right) \quad \forall h, t
$$

As for the heat pump, the following set of equations describes the considered heat pump model:

$$
\begin{array}{ll}
Q_{h, t}^{H P}=\gamma^{A R} \sum_{m} \Delta F_{h, m, t}^{H P}\left(\Gamma_{h}^{H P}-\Gamma_{h, t-1}^{R F}\right) & \forall h, t \\
\Delta F_{h, \mathrm{~m} 0, t}^{H P}=y_{h, t} \Delta \Phi_{h, \mathrm{~m} 0}^{U B} & \forall h, t \\
\Delta F_{h, m, t}^{H P} \leq y_{h, t} \Delta \Phi_{h, m}^{U B} & \forall h, m \notin\{\mathrm{m} 0\}, t \\
P_{h, t}^{H P}=\sum_{m} \beta_{m} \Delta F_{h, m, t}^{H P} & \forall h, t
\end{array}
$$


In eq. (6a), the heat flow is linked to the air heat capacity $\gamma^{A R}$ and to the difference between the output temperature of the heat pump $\Gamma_{h}^{H P}$ and the reference temperature $\Gamma_{h, t-1}^{R F}$, through the terms $\Delta F_{h, m, t}^{H P}$, which are the incremental values of air mass flow associated to the operating mode $m$ of the heat pump. When the heat pump is activated (binary status of $y_{h, t}=1$ ), the constraints given by eqs. (6b) and (6c) allow defining an integer value of incremental air mass flow $\Delta F_{h, m, t}^{H P}$ upper bounded by $\Delta \Phi_{h, m}^{U B}$. This definition permits considering a continuous operating mode of the heat pump, and in eq. (6a) the sum of all the different incremental air mass flows allows obtaining the overall air mass flow provided by the heat pump. Eq. (6d) defines instead the mapping of the air mass flow to the corresponding value of electrical power, through a coefficient $\beta_{m}$ that is the power per air mass flow of mode $m$. Through this definition of the heat pump model, the optimization algorithm defines not only the binary status of the heat pump (on or off) but also the level of operating air mass flow and, consequently, of consumed power. It is worth underlining that the exact model of the heat flow provided by the heat pump, in eq. (6a), would require to consider the difference between the heat pump output temperature and actual indoor temperature of the house. In the used model, the indoor temperature of the house has been approximated with the reference temperature selected by the customer because in this way it is possible to keep the overall model linear, achieving important computational advantages at the expenses of a slight reduction in the accuracy of the heat pump flow calculation.

\section{Evaluation of Customers Flexibility}

In the defined DSM scheme, the customers flexibility can be seen as the possibility to avoid the use of the heat pump continuously for a certain period of time or, similarly, as the amount of time for which it is possible to shift its use without losing the desired thermal comfort. A quantitative evaluation of this capability can be obtained by referring to eq. (4). When the heat pump is not operating, its heat flow is $Q_{h, t}^{H P}=0$, and (4) thus becomes:

$$
T_{h, t}^{I N}=T_{h, t-1}^{I N}-\frac{Q_{h, t}^{L S}}{\mu_{h}^{H S} \gamma^{A R}} \Delta t
$$

Substituting (5) in (7) and with few mathematical steps, it is possible to find:

$$
\Delta t=\frac{\mu_{h}^{H S} \gamma^{A R}}{\kappa_{h}^{H S}} \frac{T_{h, t-1}^{I N}-T_{h, t}^{I N}}{T_{h, t-1}^{I N}-\Gamma_{h, t-1}^{O T}}
$$

In the considered scenario, the amount of time for which the customer can shift the operation of the heat pump is maximized when the starting temperature is at the highest temperature boundary and the final one is at the lowest boundary. Considering this, the indoor temperature used for the calculation of the power losses can be set, in first approximation, equal to the average value between upper and lower boundary, which is the reference temperature chosen by the end-user. Under these assumptions, and defining the boundary temperature interval as $\Delta T_{h}=\Gamma_{h}^{U B}-\Gamma_{h}^{L B}$, it is possible to write:

$$
\Delta t_{h}^{\max }=\frac{\mu_{h}^{H S} \gamma^{A R}}{\kappa_{h}^{H S}} \frac{\Delta T_{h}}{\Gamma_{h}^{R F}-\Gamma_{h, t-1}^{O T}}
$$

Eq. (9) is an approximation of the maximum period of inactivity for the heat pump with a certain outdoor temperature and the given parameters. Four main terms affecting the customer flexibility can be identified: two of them are associated to the characteristics of the considered building and are the thermal mass of the house (given by the product $C_{h}^{H S}=\mu_{h}^{H S} \gamma^{A R}$ ) and the heat loss factor $\kappa_{h}^{H S}$; the other two are the reference temperature $\Gamma_{h}^{R F}$ and the boundary temperature interval $\Delta T_{h}$, which are decisional parameters for the end-user.

The impact of these factors on the available flexibility is easily assessable. The termal mass and the difference between the boundary temperatures are proportional to the time $\Delta t^{\max }$ and thus their increase is automatically traduced into larger flexibility. Vice versa, an increase in the heat loss factor and in the selected reference temperature leads to a decrease of the time $\Delta t^{\max }$ and thus in lower levels of flexibility. As it can be observed, customers have therefore an active role in determining their flexibility through the choice of the parameters $\Gamma_{h}^{R F}$ and $\Delta T_{h}$.

The time $\Delta t^{\max }$ can be adopted for different purposes. For example, it could be used as an index to reward differently the customers, in an incentive-based DSM program, according to the provided flexibility. Moreover, as discussed in the next Section, it can be also used to understand what kind of optimization results can be expected through the DSM scheme. This could be useful as validation of the optimization results or to refine the design of the DSM algorithm, as well as a tool, for the DSM provider, to decide the incentive strategy on the basis of the benefits potentially achievable.

Together with the flexibility in terms of time, another important parameter to quantify customers flexibility is the amount of power that can be curtailed. As indicated in Section II, customers' heat pumps can work in a given range of operating powers. To define a unique index of flexibility associated to the controllable power, the amount of power needed to compensate the heat losses of the house at a given instant of time is here chosen. Looking at eq. (4), such quantity is the electrical power needed to obtain $Q_{h, t}^{H P}=Q_{h, t}^{L S}$ or, similarly, the power required to keep the temperature inside the house at a constant value. From eq. (5) and (6a), the total air mass flow $F_{h, t}^{\text {flex }}$ equalizing the heat losses is:

$$
F_{h, t}^{\text {flex }}=\sum_{m} \Delta F_{h, m, t}^{\text {flex }}=\frac{\kappa_{h}^{H S}\left(T_{h, t-1}^{I N}-\Gamma_{h, t-1}^{O T}\right)}{\gamma^{A R}\left(\Gamma_{h}^{H P}-\Gamma_{h, t-1}^{R F}\right)}
$$

and the associated flexible power $P_{h, t}^{\text {flex }}$ results:

$$
P_{h, t}^{\text {flex }}=\sum_{m} \beta_{m} \Delta F_{h, m, t}^{\text {flex }}
$$

It is possible to observe that, in this case, the power flexibility mainly depends on the characteristics of the house 
and the heat pump. Customers settings only have a quite limited impact (given by the chosen reference temperature $\left.\Gamma_{h, t-1}^{R F}\right)$. If the air mass flow needed to compensate the heat losses is less than the minimum value allowed by the heat pump, the flexible power can be set to the minimum operating power as default value. As shown in the following, the index $P^{\text {flex }}$ can be used to assess the benefits of the DSM program in terms of power peak reduction.

\section{Estimation OF THE DSM RESUlTS USING FLEXIBILITY INDEXES}

While the analysis presented in Section III allows a straightforward evaluation of the flexibility of single customers, the proposed optimization is centralized and thus it tries to achieve the power peak minimization by considering the aggregated behaviour of all the simulated users. This leads to a more complicated scenario, because the scheduling of the different customers will be coordinated also to avoid the occurrence of other power peaks at a different time with respect to the original power peak time. Despite the complex process behind the optimization, the indexes $\Delta t^{\max }$ and $P^{\text {flex }}$ can be exploited to obtain a rough estimation of the results achievable through the DSM scheme. This can be important for a DSM provider to understand the potential benefits associated to different levels of flexibility. In the following, an iterative procedure is proposed to obtain the estimation of the expected DSM results using the presented flexibility indexes.

The fist step of the procedure is to sort the customers according to their flexibility. To this purpose, the first criterion is to rank the customers according to the maximum number of discrete time steps $T_{d}^{\max }$ for which their heat pump can be off (considering the time discretization used in the optimization algorithm). This information can be derived from $\Delta t^{\max }$ and houses with shorter $T_{d}^{\max }$ are considered as less flexible. Then, among the houses with same $T_{d}^{\max }, P^{\text {flex }}$ is used as second sorting criterion. In this case, higher values of $P^{\text {flex }}$ are associated to lower levels of flexibility. Once the houses are ranked according to flexibility, the power $P_{h}^{\text {flex }}$ of all the houses with $T_{d}^{\max }=0$ (without time flexibility) is added to the fixed power $P_{f}\left(t_{\text {peak }}\right)$ present at the peak time $t_{\text {peak }}$ to consider that these heat pumps cannot be switched off and have to work also during the peak time. In this way, a temporary value of aggregated power $P_{\text {agg }}$ is created. Then, the iterative procedure starts, aimed at considering also the other heat pumps and at creating their $\mathrm{ON}$ events. The iteration index is initially set to $n=1$. At each iteration, a time slot is added to the list of considered time slots (at the first iteration this is given by the only peak time) selecting, between the time slots adjacent to the temporary set, the one with the largest fixed power. Then, all the houses with flexibility $T_{d}^{\max } \leq n$ are considered, one by one, starting from the house with lowest flexibility, to create the heat pump ON events. For each house, a heat pump ON event is created in the time slot, among those that do not already have an ON event for that house, with the lowest aggregated power. Once the ON event is created, the aggregated power is updated by adding the power $P_{h}^{\text {flex }}$
1: Rank houses by flexibility;

2: detect power peak time $T_{\text {peak }}$;

3: put an ON event at $t=t_{\text {peak }}$ for all the HPs of the houses with $T_{d, h}^{\max }=0$;

4: update the aggregated power $P_{a g g}=P_{f}+P_{H P}$;

5: start iterative procedure: $n=1$;

6: add an adjacent time slot $t_{\text {new }}$ to the considered ones;

for $i=1$ : no. houses with $T_{d, h}^{\max } \leq n$ do

create an $\mathrm{ON}$ event for house $h_{i}$ in the time slot with

lowest aggregated power $P_{\text {agg }}$;

9: $\quad$ update aggregated power $P_{\text {agg }}$;

: end for

11: $n=n+1$

if $n \leq \max \left(T_{d, h}^{\max }\right)$ then

13: $\quad$ go back to 6 ;

4: else

15: $\quad$ repeat 6

16: $\quad$ if $P_{f}\left(t_{\text {new }}\right)>P_{\text {agg }}\left(t_{\text {peak }}\right)-\sum_{h} P_{h}^{\text {flex }}$ then

17: $\quad$ go back to 7 ;

18: $\quad$ else

19: $\quad$ stop the procedure;

20: $\quad$ end if

1 : end if

Fig. 1. Procedure for DSM results estimation

of the considered heat pump to the selected time slot. This procedure has to run for all the houses with $T_{d}^{\max } \leq n$ and, when finished, a new iteration with $n=n+1$ starts. The overall iterative procedure stops if all the houses have been considered and when, at the beginning of the new iteration, the adjacent time slot to be considered has a fixed power $P_{f}\left(t_{\text {new }}\right) \leq P_{\text {agg }}\left(t_{\text {agg,peak }}\right)-\sum_{h} P_{h}^{\text {flex }}$, which means that the new considered time slot has a fixed power sufficiently low to allocate the heat pump power $P^{\text {flex }}$ of all the houses without creating a new power peak.

This procedure, which is summarized in Fig. 1, does not allow to build the real scheduling of the heat pumps; however, it permits to obtain a rough idea about the number of heat pumps that are expected to work during the peak time and about the level of additional power that will be allocated on top of the fixed load. In fact, with the proposed procedure, each heat pump keeps the temperature constant during time slots with $\mathrm{ON}$ operation (consuming the power $P^{\text {flex }}$ ) and has a number of OFF periods equal to the available discretized time flexibility $T_{d}^{\max }$. The iterative procedure and the update of the aggregated power $P_{\text {agg }}$ at each heat pump event creation allow considering the possible generation of new power peaks in the time slots close to the original power peak time and thus implement a logic similar to the one used by the optimization algorithm. Therefore, under the assumption that the indoor temperature was risen to its maximum boundary during a previous off-peak period, this procedure allows fully exploiting the flexibility made available by each house, while considering the constraints brought by the centralized approach, which operates in order also to avoid the creation of other power 
peaks in the time slots adjacent to the original power peak time. In the next Section, the results of this procedure, based on the use of the proposed flexibility indexes, will be compared to those given by the optimization algorithm in order to assess the goodness of such estimation.

\section{Simulations And Results}

To assess the impact of the different factors affecting the customers' flexibility and to evaluate the goodness of the proposed procedure for estimating the DSM results, simulations have been run considering a scenario of 240 houses, among which 60 houses have been supposed to be equipped with electrical heat pumps. The fixed load considered for the 240 houses was extracted using statistical data related to typical residential power profiles. In the considered scenario the power peak time is at 20:00. The main goal of the simulations is to assess the impact of the customers' decisional parameters on their flexibility and, consequently, on the results of the DSM scheme. For this reason, and for the sake of simplicity, all the houses were chosen with identical characteristics and only the reference temperature or the temperature boundaries were changed to analyse different cases. In particular, an heat loss factor $\kappa^{H S}$ equal to $63.4 \mathrm{~W} /{ }^{\circ} \mathrm{C}$ and an indoor air mass of the houses of $1823 \mathrm{~kg}$ were used in all the simulations.

The first simulation aims at investigating the impact of different temperature boundaries. To this purpose, a reference temperature equal to $21^{\circ} \mathrm{C}$ was chosen and temperature boundaries $\Delta T_{h}$ equal to $1^{\circ} \mathrm{C}, 2^{\circ} \mathrm{C}$ and $3^{\circ} \mathrm{C}$ were tested. Through the use of these parameters, the corresponding time flexibility $\Delta t^{\max }$ calculated at the peak time (for an outdoor temperature of $5^{\circ} \mathrm{C}$ ) is slightly larger than 30,60 and 90 minutes (for the increasing values of temperature boundaries). The results of aggregated power at grid level, obtained through the optimization algorithm, are shown in Fig. 2. The impact of the customers flexibility can be clearly evaluated looking at the peak and off-peak periods. It is possible to observe that, for increasing levels of flexibility, the power added on top of the peaks decreases while more power is consumed during off-peaks periods to charge the house with thermal energy. In particular, when having low flexibility $\left(\Delta T_{h}=1{ }^{\circ} C\right)$, the optimization schedules 41 heat pumps (among the 60 houses with electric heating) to run during the peak time, bringing an additional power of more than $16 \mathrm{~kW}\left(P^{\text {flex }}\right.$ is in this case the

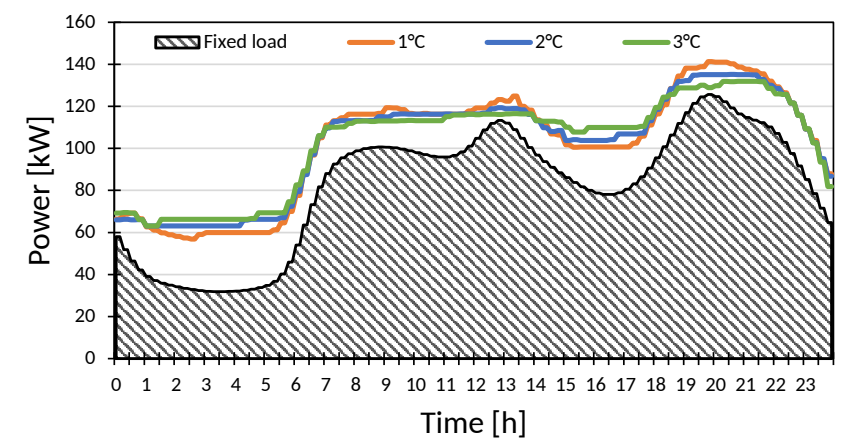

Fig. 2. Aggregated power in the grid with changing temperature boundaries.
TABLE I

OPERATING HeAt PuMPS With DifFERENT FLEXIBILITy LEVELS

\begin{tabular}{c|c|c|c|c|c}
\hline \multirow{2}{*}{ Case } & \multicolumn{5}{|c}{ Time slot } \\
\cline { 2 - 6 } & $19: 30$ & $19: 45$ & $20: 00$ & $20: 15$ & $20: 30$ \\
\hline$\Delta t_{h}=1^{\circ} C$ & & & & & \\
Optimization & 35 & 39 & 41 & 47 & 52 \\
Estimation & 46 & 37 & 33 & 35 & 44 \\
\hline$\Delta t_{h}=2^{\circ} C$ & & & & & \\
Optimization & 33 & 26 & 23 & 26 & 32 \\
Estimation & 32 & 25 & 22 & 25 & 31 \\
\hline$\Delta t_{h}=3^{\circ} C$ & & & & & \\
Optimization & 19 & 14 & 10 & 16 & 25 \\
Estimation & 24 & 16 & 13 & 16 & 22 \\
\hline
\end{tabular}

minimum operating power of the heat pump, which is equal to $400 \mathrm{~W}$ for all the customers). When having larger flexibility $\left(\Delta T_{h}=3^{\circ} \mathrm{C}\right)$, instead, only 10 houses have an operating heat pump at 20:00. The case with $\Delta t_{h}=2^{\circ} \mathrm{C}$ gives an intermediate result, with 23 heat pumps scheduled to run at the peak time.

The results given by the DSM optimization have been compared to those obtained through the estimation procedure presented in Section IV. Table I shows the heat pumps scheduled to be $\mathrm{ON}$ according to the optimization algorithm and those allocated by the estimation procedure during the fixed load peak time (20:00) and the close time slots (the considered time discretization is 15 minutes). In the case with $\Delta T_{h}=1{ }^{\circ} \mathrm{C}$, some differences have been found between the two solutions. However, looking at the distribution of the heat pumps scheduled to work according to the DSM optimization, it is possible to observe that a lower number of heat pumps is $\mathrm{ON}$ in the time slots before the peak time. This suggests that the found solution is not the global optimum (all the optimizations stop after a maximum time of two hours) and, in fact, when checking the scheduling of the single customers, some houses are found where the heat pump operation could be shifted from the peak time to the time slots immediately before the peak without violating the thermal constraints (thus decreasing the power peak). In case of $\Delta T_{h}=2^{\circ} C$, the results of the estimation procedure are very close to those of the optimization. Referring to Fig. 2, it is possible to note that in this case the profile of aggregated power is very flat around the peak time. This indicates that the operation of the heat pumps given by the optimization is well distributed in order to fulfil the goal of minimizing the power peak and thus the solution is probably close to the global optimum. Finally, in the case with $\Delta T_{h}=3^{\circ} \mathrm{C}$, the results are quite close even if they do not perfectly match. The slight differences could be either due to the non-global optimum solution found by the DSM optimization or because of the simplifications intrinsically considered in the estimation procedure.

In general, however, the outcomes show that the proposed flexibility indexes could be helpful, together with the estimation tool, to have a rough prediction of the possible DSM results and also to refine non optimal solutions found by the DSM optimization. In terms of power peak reduction (with 
respect to a reference scenario where all the heat pumps are consuming $P^{\text {flex }}$ at the peak time), the DSM optimization allows cutting $5.6 \%, 9.7 \%$ and $11.9 \%$ of the peak power (for the increasing values of $\Delta T_{h}$, respectively). The simplified procedure allows obtaining similar results and estimates a potential of $7.2 \%, 10.2 \%$ and $12.6 \%$ power peak reduction. The analysis of this trend for different levels of customer flexibility can be important to justify the use of a DSM scheme in a particular scenario or, for example, to suitably choose the incentives for the customers in order to obtain the desired level of flexibility.

A further simulation was run to consider the variations on the other decisional parameter of the end-users, namely the indoor reference temperature. In this case, the temperature boundary interval was fixed to $2^{\circ} \mathrm{C}$ while the reference temperature was changed to $20^{\circ} \mathrm{C}, 21^{\circ} \mathrm{C}$ and $22^{\circ} \mathrm{C}$ in the different tests. As suggested by eq. (9), higher reference temperatures lead to a decreased flexibility. However, changes in the order of few degrees do not bring large differences. In fact, in the considered scenario, the corresponding values of $\Delta t^{\max }$ only differ of 4 minutes when increasing the reference temperature of $1^{\circ} \mathrm{C}$. The small differences predicted through the evaluation of the index $\Delta t^{\max }$ are also confirmed looking at the results shown in Fig. 3. In this case, slight differences are present among the different cases around the peak time. As shown in Fig. 3, the main difference is on the total energy needed to provide the desired thermal comfort. However, the larger demand brought by higher reference temperatures is mainly fulfilled during the off-peak hours and the power demand during peak hours is only slightly affected. The power peak reduction obtained through the DSM optimization is $10.2 \%$, $9.7 \%$ and $7.8 \%$ for the increasing values of reference temperature. The motivation for the larger step when moving to a reference temperature equal to $22^{\circ} \mathrm{C}$ is the decrease of $\Delta t^{\max }$ below 1 hour. In fact, due to the 15 minutes time discretization in the optimization, such a reduction implies that the maximum number of discrete time steps for which the heat pumps can be off (without violating the thermal comfort requirements of the customers) is three. This aspect is also confirmed looking at the individual scheduling of the single customers, where the heat pumps are off for a maximum of three time steps around the peak period. When evaluating the power peak reduction with the estimation tool, taking into account $T_{d}^{\max }=3$, a potential power reduction equal to $8.5 \%$ is found, which is indeed quite close to the found result.

\section{CONCLUSION}

In this paper, an analysis of the impact of the customers flexibility on the results of an optimization algorithm designed to achieve power peak shaving in the grid through the scheduling of electric heat pumps has been presented. The performed analysis allows to define, from a quantitative point of view, end-users flexibility in terms of time (how long the heat pumps can be turned off to alleviate the power demand during peak hours) and power (how much power can be expected to be curtailed thanks to the provided flexibility). The proposed

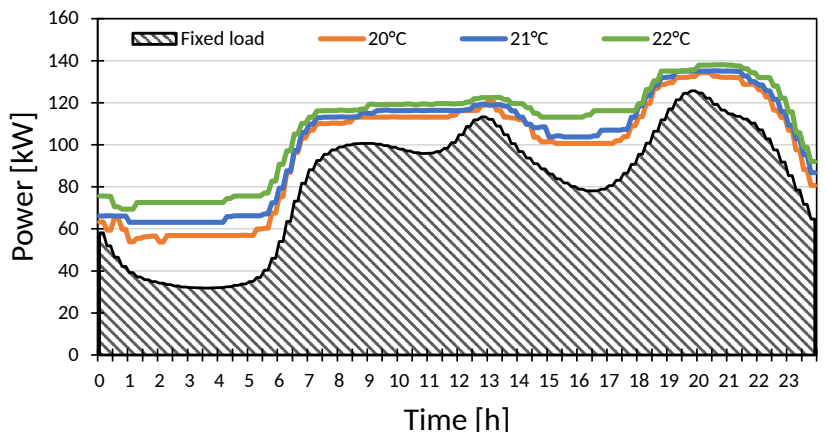

Fig. 3. Aggregated power in the grid with changing reference temperature.

indexes also allow for a rough estimation of the expected benefits given by the presented DSM scheme, which can be helpful to evaluate the need for a DSM program or to suitably choose the incentives for the customers.

\section{ACKNOWLEDGMENT}

This work was supported by FLEXMETER, which is an EU Horizon 2020 project under grant agreeement no. 646568.

\section{REFERENCES}

[1] J. Fan and S. Borlase, "The evolution of distribution," IEEE Power and Energy Magazine, vol. 7, no. 2, pp. 63-68, March 2009.

[2] J. Barton, S. Huang, D. Infield, M. Leach, D. Ogunkunle, J. Torriti, and M. Thomson, "The evolution of electricity demand and the role for demand side participation, in buildings and transport," Energy Policy, vol. 52, pp. 85-102, 2013.

[3] P. Palensky and D. Dietrich, "Demand side management: Demand response, intelligent energy systems, and smart loads," IEEE Transactions on Industrial Informatics, vol. 7, no. 3, pp. 381-388, Aug 2011.

[4] D. Papadaskalopoulos and G. Strbac, "Decentralized participation of flexible demand in electricity markets - part i: Market mechanism," IEEE Transactions on Power Systems, vol. 28, no. 4, pp. 3658-3666, Nov 2013.

[5] F. Rahimi and A. Ipakchi, "Demand response as a market resource under the smart grid paradigm," IEEE Transactions on Smart Grid, vol. 1, no. 1, pp. 82-88, June 2010.

[6] V. S. K. M. Balijepalli, V. Pradhan, S. A. Khaparde, and R. M. Shereef, "Review of demand response under smart grid paradigm," in ISGT2011 India, Dec 2011, pp. 236-243.

[7] M. H. Albadi and E. F. El-Saadany, "Demand response in electricity markets: An overview," in 2007 IEEE Power Engineering Society General Meeting, June 2007, pp. 1-5.

[8] G. Strbac, "Demand side management: Benefits and challenges," Energy Policy, vol. 36, no. 12, pp. 4419-4426, 2008, foresight Sustainable Energy Management and the Built Environment Project.

[9] N. Chapman, L. Zhang, N. Good, and P. Mancarella, "Exploring flexibility of aggregated residential electric heat pumps," in 2016 IEEE International Energy Conference (ENERGYCON), April 2016, pp. 1-6.

[10] B. P. Bhattarai, B. Bak-Jensen, J. R. Pillai, and M. Maier, "Demand flexibility from residential heat pump," in 2014 IEEE PES General Meeting - Conference Exposition, July 2014, pp. 1-5.

[11] A. Arteconi, N. Hewitt, and F. Polonara, "Domestic demand-side management (dsm): Role of heat pumps and thermal energy storage (tes) systems," Applied Thermal Engineering, vol. 51, no. 1, pp. 155-165, 2013.

[12] F. De Angelis, M. Boaro, D. Fuselli, S. Squartini, F. Piazza, and Q. Wei, "Optimal home energy management under dynamic electrical and thermal constraints," Industrial Informatics, IEEE Transactions on, vol. 9, no. 3, pp. 1518-1527, 2013.

[13] K. M. Nielsen, T. S. Pedersen, and P. Andersen, "Heat pumps in private residences used for grid balancing by demand desponse methods," in 2012 IEEE PES Transmission and Distribution Conference and Exposition, May 2012, pp. 1-6. 
[14] Z. Csetvei, J. Østergaard, and P. Nyeng, "Controlling price-responsive heat pumps for overload elimination in distribution systems," in 2011 2nd IEEE PES International Conference and Exhibition on Innovative Smart Grid Technologies, Dec 2011, pp. 1-8.

[15] J. L. Cremer, M. Pau, F. Ponci, and A. Monti, "Optimal scheduling of heat pumps for power peak shaving and customers thermal comfort," in Proceedings of the 6th International Conference on Smart Cities and Green ICT Systems, Apr 2017, pp. 23-34. 